\title{
DIMENSION OF CHARACTER VARIETIES FOR 3-MANIFOLDS
}

\author{
E. FALBEL AND A. GUILLOUX \\ (Communicated by Michael Wolf)
}

\begin{abstract}
Let $M$ be an orientable 3-manifold, compact with boundary and $\Gamma$ its fundamental group. Consider a complex reductive algebraic group $G$. The character variety $X(\Gamma, G)$ is the $\operatorname{GIT}$ quotient $\operatorname{Hom}(\Gamma, G) / / G$ of the space of morphisms $\Gamma \rightarrow G$ by the natural action by conjugation of $G$. In the case $G=\mathrm{SL}(2, \mathbb{C})$ this space has been thoroughly studied.

Following work of Thurston (1980), as presented by Culler-Shalen (1983), we give a lower bound for the dimension of irreducible components of $X(\Gamma, G)$ in terms of the Euler characteristic $\chi(M)$ of $M$, the number $t$ of torus boundary components of $M$, the dimension $d$ and the rank $r$ of $G$. Indeed, under mild assumptions on an irreducible component $X_{0}$ of $X(\Gamma, G)$, we prove the inequality
\end{abstract}

$$
\operatorname{dim}\left(X_{0}\right) \geq t \cdot r-d \chi(M)
$$

\section{INTRODUCTION}

Representation varieties of fundamental groups of 3-manifolds have been studied for a long time. They are important, on one hand, to understand geometric structures on 3-manifolds and their topology as Thurston's work 21] has shown and, on the other hand, to obtain more refined topological information on 3-manifolds as in the Culler-Shalen theory [4].

The dimension of the character variety of $\pi_{1}(M)$ on $\operatorname{PSL}(2, \mathbb{C})$ (where $M$ is a cusped hyperbolic manifold) at the representation corresponding to a complete finite volume hyperbolic structure is exactly the number of cusps (see [17] where it is shown that, in fact, that representation is a smooth point of the character variety). A general bound was established by Thurston and made more precise by Culler-Shalen: the dimension of irreducible components containing irreducible representations with non-trivial boundary holonomy is bounded below by the number of cusps. In fact there are examples of irreducible representations with arbitrary large dimensions associated to hyperbolic manifolds with even only one cusp.

The proof of the bound given by both Thurston and Culler-Shalen uses trace identities for $\operatorname{SL}(2, \mathbb{C})$ which are difficult to work with in the higher dimensional case.

In this paper we obtain a bound on the dimension of irreducible components which contain representations satisfying reasonable genericity conditions. They include a notion of irreducibility and one of boundary regularity. The bound is expressed in terms of the Euler characteristic $\chi(M)$ of $M$, the number $t$ of torus

Received by the editors December 15, 2015 and, in revised form, April 6, 2016.

2010 Mathematics Subject Classification. Primary 57M27.

This work was supported in part by the ANR through the project "Structures Géométriques et Triangulations". 
boundary components of $M$, the dimension $d$ and the rank $r$ of $G$. Indeed, for an irreducible component $X_{0}$ of $X(\Gamma, G)$ satisfying the assumptions, we prove the inequality

$$
\operatorname{dim}\left(X_{0}\right) \geq t \cdot r-d \chi(M) .
$$

In the case $G=\operatorname{SL}(n, \mathbb{C})$, thoroughly discussed below, the component containing the geometric representation in $\operatorname{SL}(n, \mathbb{C})$ has the minimal bound in our estimate (see for example [15]). But we don't know if our bound is sharp for other reductive groups.

An important remark is that the bound obtained may be described as half the dimension of the character variety of $\partial M$. This is directly related to a description of the image (under restriction) of $X_{0}$ in the character variety of $\partial M$. In certain cases this image is a Lagrangian submanifold, as discussed in [19, Thm. 61].

In Section 1 we explain our result for $\operatorname{SL}(n, \mathbb{C})$. Although a particular case of our general result, it is simpler to state and contains the essential idea. We prove the bound for any component containing a representation satisfying two conditions. We assume that its image is Zariski-dense and that the image of the fundamental group of the boundary is regular. The latter means that each image of a boundary fundamental group has a centralizer of minimal dimension (equal to the rank $n-1$ of $\operatorname{SL}(n, \mathbb{C}))$. Note that the boundary regularity condition in the $\operatorname{SL}(2, \mathbb{C})$ case simply means that the image is not central for each boundary component.

We consider next representations into complex reductive affine algebraic groups. We use the general definitions of irreducibility as in Sikora [19] and the notion of regularity in reductive groups following Steinberg [20] and prove the main Theorem 5.

A special case of the theorem occurs when $M$ is an oriented complete cusped hyperbolic manifold and $\rho: \pi_{1}(M) \rightarrow \mathrm{SL}(n, \mathbb{C})$ is the representation obtained by composing a representation $\pi_{1}(M) \rightarrow \mathrm{SL}(2, \mathbb{C})$ obtained from the hyperbolic structure with the irreducible embedding $\mathrm{SL}(2, \mathbb{C}) \rightarrow \mathrm{SL}(n, \mathbb{C})$. That representation is called a geometric representation and the dimension of the irreducible component of the character variety $X(\Gamma, \operatorname{SL}(n, \mathbb{C}))$ containing it was first described in [15] (see also [3, 10]). Evidence that this bound was valid for all components containing irreducibles was obtained in computations of the character variety for the figure eight knot fundamental group into $\mathrm{SL}(3, \mathbb{C})$ [6, 12. In this case, irreducible components containing irreducible representations are all two dimensional. Moreover each component contains Zariski-dense and boundary regular unipotent representations already computed in [5] (see also [1,7] for other examples).

One can use the results on local rigidity 3 in order to prove that a component seen by the Deformation variety of an ideal triangulation (defined in [3]) verifies this bound. We do not discuss this here, going straight to the proof which does not use triangulations.

As a final remark, we should note that an a priori bound on the dimension of irreducible components certainly simplifies effective computations as in [6] by eliminating the need to check the existence of lower dimensional components.

\section{A SIMPle CASE}

Before going to precise definitions in the framework of algebraic groups, let us sketch the proof in a simple yet interesting case. Namely, in this section we 
assume $G=\operatorname{SL}(n, \mathbb{C})$. It contains the original statement of Thurston (for the group $\operatorname{SL}(2, \mathbb{C}))$. Recall that its dimension $d$ is $n^{2}-1$ and its rank $r$ is $n-1$ : this is the minimal dimension of the centralizer of an element.

Let $M$ be an orientable 3-manifold, compact with boundary. In the following we suppose that no boundary component is a 2-sphere. Although the bound we obtain is valid in this case, if we simply fill the 2 -sphere with a ball we obtain a better estimate. Let $t$ be the number of torus boundary components. We fix for each torus boundary component an injection (denoted by an inclusion) $\pi_{1}(T) \subset \Gamma$. In other words, for each torus boundary component, we choose a lift in the universal covering of $M$.

We want a lower bound on the dimension of components of

$$
X(\Gamma, G)=\operatorname{Hom}(\Gamma, G) / / G \text {. }
$$

So consider an irreducible component $R_{0}$ of $\operatorname{Hom}(\Gamma, G)$ and $X_{0}$ its projection in $X(\Gamma, G)$. We work with two assumptions on an element $\rho_{0} \in R_{0}$. One assumption should be a form of irreducibility for the whole representation $\rho_{0}$. The second assumption is a regularity assumption for the image under $\rho_{0}$ of the fundamental groups of the torus boundaries. In the first version of our theorem, we assume:

- Zariski-density: $\rho_{0}(\Gamma)$ is Zariski-dense in $G$.

- Boundary regularity: For any torus boundary component $T \subset \partial M, \rho_{0}$ maps $\pi_{1}(T)$ to a regular subgroup of $\operatorname{SL}(n, \mathbb{C})$, i.e., every subspace on which elements in $\rho_{0}\left(\pi_{1}(T)\right)$ act by homothety is a line.

The second assumption is for example satisfied if the image of $\pi_{1}(T)$ is diagonalizable and every global eigenspace is a line, or if this image is unipotent and fixes a unique flag. It implies that the number of invariant subspaces is finite. It implies moreover that the centralizer of the image of $\pi_{1}(T)$ has dimension $n-1$.

We get the following particular case of the general theorem:

Theorem 1. Let $X_{0}$ be the projection in $X(\Gamma, G)$ of an irreducible component $R_{0}$ of $\operatorname{Hom}(\Gamma, G)$ containing a representation $\rho_{0}$ which is Zariski-dense and boundary regular.

Then we have:

$$
\operatorname{dim}\left(X_{0}\right) \geq(n-1) t-\left(n^{2}-1\right) \chi(M) .
$$

Note that the case of $\rho_{0}$ being the geometric representation is not handled by this theorem, as the representation is not Zariski-dense. It will be handled by the general case; see Theorem 5 .

Proof. Let us sketch the proof, inspired by Thurston and Culler-Shalen 4]. It works by induction on the number $t$ of boundary tori.

Initialization: $t=0$. The inequality $\operatorname{dim}\left(X_{0}\right) \geq-\left(n^{2}-1\right) \chi(M)$ in this case is very general and was already known by Thurston. We give a proof in Section 3.2 . Note that if $\chi(M)$ is non-negative the formula does not carry any information.

Propagation: $t-1 \rightarrow t$. Let $T$ be a torus boundary in $\partial M$. We first use

Lemma 2. There exists an element $\gamma$ of $\Gamma$ such that $\rho_{0}\left(\pi_{1}(T)\right)$ and $\rho_{0}(\gamma)$ generate an irreducible representation of the subgroup of $\Gamma$ generated by $\pi_{1}(T)$ and $\gamma$.

Proof. By the boundary regular assumption, $\rho_{0}\left(\pi_{1}(T)\right)$ has a finite number of stable subspaces. By Zariski-density of $\rho_{0}(\Gamma)$, we find an element $\gamma \in \Gamma$ such that $\rho_{0}(\gamma)$ does not stabilize any of these subspaces. Indeed, the union of all elements in the 


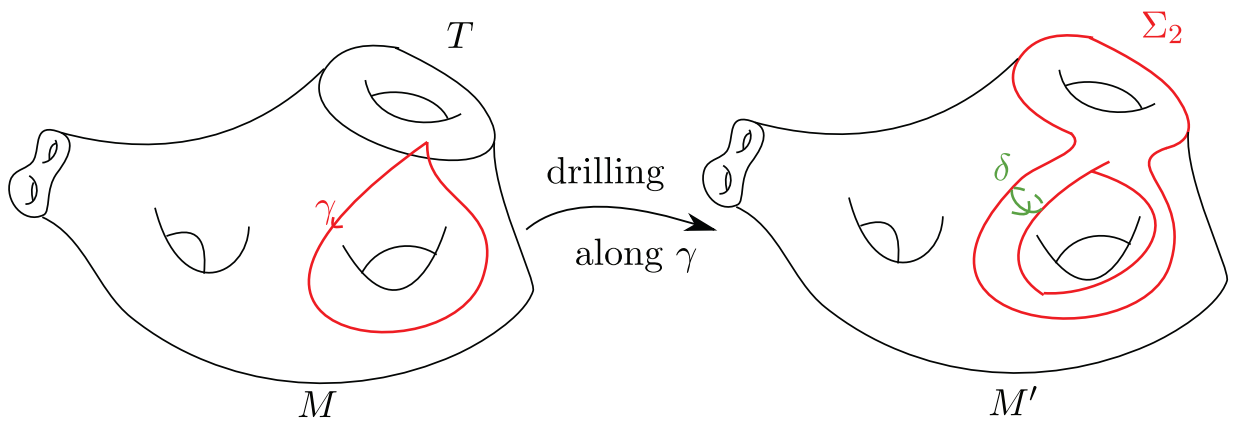

Figure 1. Drilling along $\gamma$

image of $\rho$ which preserves one of the subspaces is contained in a Zariski closed subset.

Hence $\rho_{0}(\gamma)$ and $\rho_{0}\left(\pi_{1}(T)\right)$ spans an irreducible subgroup of $G$.

Remark 1.1. Using section 4.4 in [22], one may prove the above lemma (and then the theorem) with a mildest irreducibility assumption and a different kind of regularity: namely that the Zariski-closure of $\rho_{0}$ is almost simple and irreducible, as e.g. the image of $S L_{2}(\mathbb{C})$ under the irreducible representation and the boundary torus is mapped on a non-trivial diagonal subgroup. This would be applicable to the geometric representation. However we will not precisely discuss this, as this will be handled by the general theorem.

Now, following Thurston and 4, we drill along $\gamma$ in $M$. We get a new 3-manifold $M^{\prime}$ with a new genus 2 boundary component denoted by $\Sigma_{2}$ and $t-1$ torus boundary components. Denote by $\Gamma^{\prime}$ its fundamental group. Let $\delta$ be a meridian of the new handle of $\Sigma_{2}$ ( $\delta$ is an element of $\pi_{1}\left(\Sigma_{2}\right)$ whose normal closure $N \Gamma^{\prime}(\delta)$ in $\Gamma^{\prime}$ is the kernel of the map $\left.\Gamma^{\prime} \rightarrow \Gamma\right)$. We have an inclusion $\pi_{1}\left(\Sigma_{2}\right) \subset \Gamma^{\prime}$ and a surjective homomorphism $\Gamma^{\prime} \rightarrow \Gamma$. Summarizing:

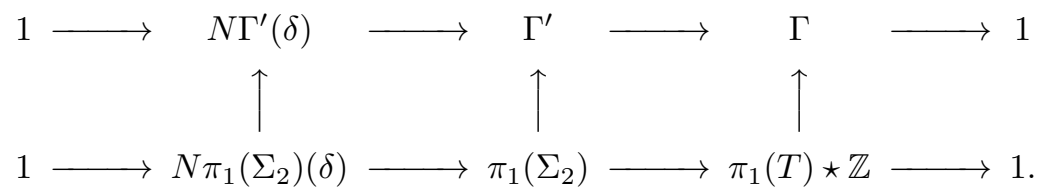

We obtain therefore a natural injection $\operatorname{Hom}(\Gamma, \operatorname{SL}(n, \mathbb{C})) \hookrightarrow \operatorname{Hom}\left(\Gamma^{\prime}, \operatorname{SL}(n, \mathbb{C})\right)$, that we see as an inclusion. Let $V \subset \operatorname{Hom}\left(\Gamma^{\prime}, G\right)$ be an irreducible component containing $R_{0}$. Then $R_{0}$ is an irreducible component of the subvariety:

$$
\{\rho \in V \mid \rho(\delta)=1\} .
$$

Let Res be the restriction map:

$$
\text { Res : } \operatorname{Hom}\left(\Gamma^{\prime}, \operatorname{SL}(n, \mathbb{C})\right) \rightarrow \operatorname{Hom}\left(\pi_{1}\left(\Sigma_{2}\right), \operatorname{SL}(n, \mathbb{C})\right) \text {. }
$$

We rephrase the previous remark on $R_{0}$ by saying that it is a component (in the variety $V$ ) of the preimage of the subvariety

$$
S=\left\{\tau \in \operatorname{Hom}\left(\pi_{1}\left(\Sigma_{2}\right), \operatorname{SL}(n, \mathbb{C})\right) \mid \tau(\delta)=1\right\}
$$


of the variety $V^{\prime}=\operatorname{Hom}\left(\pi_{1}\left(\Sigma_{2}\right), \mathrm{SL}(n, \mathbb{C})\right)$ by the regular map Res. We summarize the construction in the following maps:

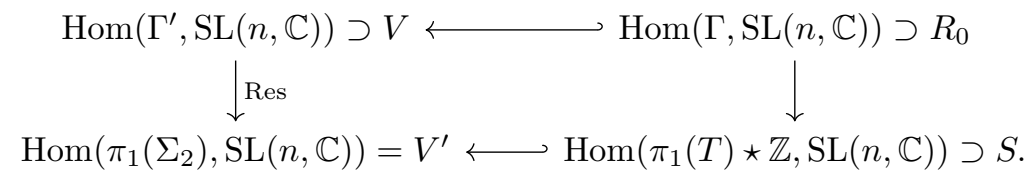

The following lemma is not difficult to prove that under our hypotheses, using fundamental results on the character varieties of surface groups (see Theorem 4). A general version of this lemma will be stated in Section 2.1.2.

Lemma 3. The codimension of $S$ in $V^{\prime}$ is $n^{2}-n$. Moreover $\operatorname{Res}\left(\rho_{0}\right)$ is a smooth point of both $S$ and $V^{\prime}$.

Proof. Let $\tau_{0}=\operatorname{Res}\left(\rho_{0}\right)$. Observe first that $\tau_{0}$ is irreducible, hence a smooth point. The dimension of $V^{\prime}$ at $\tau_{0}$ is $3\left(n^{2}-1\right)$.

Secondly, the dimension of $S$ equals the dimension of $\operatorname{Hom}\left(\pi_{1}(T), \operatorname{SL}(n, \mathbb{C})\right)$, which is $n^{2}+n-2$ (here we use the regularity hypothesis: the dimension of the centralizer of $\tau_{0}\left(\pi_{1}(T)\right)$ is the rank $\left.r=n-1\right)$ plus the dimension of the representations of $\langle\gamma\rangle$ which is $n^{2}-1$. As it is the minimal possible dimension (again by the regularity hypothesis), it is a smooth point.

Computing the difference, we get $n^{2}-n$.

A crucial yet elementary point is the following proposition. See 16. Theorem 2 , p. 48] for a related result, but we did not find the following version and therefore include a proof of it. The smoothness hypothesis in the proposition is essential, otherwise one needs other assumptions.

Proposition 1. Let $f: V \rightarrow V^{\prime}$ be a regular map between irreducibe algebraic varieties and $S \subset V^{\prime}$ a subvariety. Let $p \in V$ be such that $f(p)$ belongs to $S$ and moreover is smooth for both $S$ and $V^{\prime}$. Let $\operatorname{codim}(S)$ be the maximal codimension of an irreducible component of $S$ in $V^{\prime}$.

Then, any irreducible component of $f^{-1}(S)$ containing $p$ has codimension at most $\operatorname{codim}(S)$.

Proof. Let $C$ be a component of $f^{-1}(S)$ containing $p$. Up to restricting to an irreducible component of $S$ containing $f(C)$, we may assume $S$ to be irreducible.

We note that the set $C_{\text {good }}$ of points $p$ in $C$ whose image is a smooth point of both $S$ and $V^{\prime}$ is Zariski-open. Indeed, the sets $V_{\text {smooth }}^{\prime}$ and $S_{\text {smooth }}$ are Zariski-open subsets respectively of $V^{\prime}$ and $S$. In turn, $S \cap V_{\text {smooth }}^{\prime}$ is a Zariski-open subset of $S$. Hence, the set $S_{\text {good }}=S_{\text {smooth }} \cap V_{\text {smooth }}^{\prime}$ is a Zariski-open subset of $S$. Taking its preimage by $f$, we get the subset $C_{\text {good }}=f^{-1}\left(S_{\text {good }}\right)$ which becomes Zariski-open in $C$.

Moreover, $C_{\text {good }}$ is not empty as it contains the point $p$. As the subset $C_{\text {smooth }}$ of smooth points of $C$ is another non-empty Zariski-open subset of $C$, we conclude that they intersect non-trivially. So, up to changing the point $p$ for a point in this intersection, we may assume that $C$ is smooth at $p$. 
The map $f$ induces a Zariski tangent map $T_{p} f$ from $T_{p} V$ to $T_{f(p)} V^{\prime}$ such that $T_{p} C=T_{p} f^{-1}\left(T_{f(p)}(S)\right)$. This linear map $T_{p} f$ induces in turn an injective map from $T_{p} V / T_{p} C$ to $T_{f(p)} V^{\prime} / T_{f(p)} S$. We deduce that:

$$
\operatorname{codim}_{T_{p} V}\left(T_{p} C\right) \leq \operatorname{codim}_{T_{f(p)} V^{\prime}}\left(T_{f(p)} S\right) .
$$

Now, from the smoothness considerations we made, we get the equalities:

$$
\operatorname{dim}\left(T_{p} C\right)=\operatorname{dim}(C), \quad \operatorname{dim}\left(T_{f(p)} S\right)=\operatorname{dim}(S) \text { and } \operatorname{dim}\left(T_{f(p)} V^{\prime}\right)=\operatorname{dim}\left(V^{\prime}\right) .
$$

We do not have any smoothness information about $p$ inside $V$, but we still have the general property that $\operatorname{dim}\left(T_{p} V\right) \geq \operatorname{dim}(V)$.

Eventually, we get the desired inequality:

$$
\operatorname{codim}_{V}(C) \leq \operatorname{codim}_{T_{p} V}\left(T_{p} C\right) \leq \operatorname{codim}_{T_{f(p)} V^{\prime}}\left(T_{f(p)} S\right)=\operatorname{codim}_{V^{\prime}} S .
$$

As a corollary, using Proposition 1 we conclude that the codimension of $R_{0}$ in $V$ is at most $n^{2}-n$.

By induction (we are working at the level of representation variety, so there is an additional $\left.n^{2}-1\right)$ :

$$
\operatorname{dim}(V) \geq(n-1)(t-1)-\left(n^{2}-1\right)\left(\chi\left(M^{\prime}\right)-1\right)
$$

and therefore

$$
\operatorname{dim}(V) \geq(n-1) t-\left(n^{2}-1\right)(\chi(M)-1)+n^{2}-n .
$$

The upper bound for the codimension of $R_{0}$ grants that:

$$
\operatorname{dim}\left(R_{0}\right) \geq(n-1) t-\left(n^{2}-1\right)(\chi(M)-1) .
$$

Now projecting to $X(\Gamma, G)$, we lose $n^{2}-1$ dimensions (as $\rho_{0}$ is irreducible):

$$
\operatorname{dim}\left(X_{0}\right) \geq(n-1) t-\left(n^{2}-1\right) \chi(M) .
$$

\section{BACKGROUND ON CHARACTER VARIETIES AND REDUCTIVE GROUPS}

We now proceed with the general result, beginning with some definitions and results on algebraic groups and regularity, character varieties and irreducibility and character varieties of surface groups. This will lead to a general theorem whose proof is essentially the same as before. Let $G$ be an affine reductive algebraic group of dimension $d$ and whose center has dimension $z$. Suppose $\Gamma$ is a finitely generated group.

2.1. Character and representation varieties. Our reference for character varieties is the paper of Sikora [19]. 
2.1.1. Generalities. The set of all homomorphisms $\operatorname{Hom}(\Gamma, G)$ is an affine variety. The action of $G$ on $\operatorname{Hom}(\Gamma, G)$ is algebraic but the set theoretical quotient $\operatorname{Hom}(\Gamma, G) / G$ is not necessarily an affine variety. For that reason one works with the categorical quotient $\operatorname{Hom}(\Gamma, G) / / G$. It is an affine variety such that the projection

$$
\operatorname{Hom}(\Gamma, G) \rightarrow \operatorname{Hom}(\Gamma, G) / / G
$$

is constant on $G$-orbits and such that any morphism from $\operatorname{Hom}(\Gamma, G)$ to an algebraic variety, which is constant on orbits, factors through the projection. It exists with our hypothesis that $G$ is reductive.

Remark 2.1. Let $R_{0}$ be an irreducible component of $\operatorname{Hom}(\Gamma, G)$ and $X_{0}$ its projection to $X(\Gamma, G)$. At a generic point $\rho$, one may compute the dimension of $R_{0}$ and $X(\Gamma, G)$ as the dimension of the Zariski tangent space. Let $Z(\rho)$ be the centralizer in $G$ of $\rho(\Gamma)$. It is the subgroup of $G$ fixing $\rho$ when acting by conjugation. We have then that $\operatorname{dim}\left(X_{0}\right)=\operatorname{dim}\left(R_{0}\right)-(\operatorname{dim}(G)-\operatorname{dim}(Z(\rho))$.

In any case, we have

$$
\operatorname{dim}\left(X_{0}\right) \geq \operatorname{dim}\left(R_{0}\right)-d+z .
$$

Remark 2.2. It is known that $\operatorname{Hom}(\mathbb{Z} \oplus \mathbb{Z}, \mathrm{SL}(n, \mathbb{C}))$ is an irreducible variety 18 , Theorem C]. Also, as $\operatorname{Hom}\left(\Gamma_{1} \star \Gamma_{2}, G\right)=\operatorname{Hom}\left(\Gamma_{1}, G\right) \times \operatorname{Hom}\left(\Gamma_{2}, G\right), \operatorname{Hom}((\mathbb{Z} \oplus \mathbb{Z}) \star$ $\mathbb{Z}, \mathrm{SL}(n, \mathbb{C}))$ is an irreducible variety.

2.1.2. Dimension of representation varieties for surface groups. The most studied representation variety is the case where $\Gamma$ is a surface group. In particular, dimensions of character varieties were computed by Weil ([23]). We refer to Goldman's paper for a modern presentation.

Theorem 4 (Thm 1.2 in [8]). Let $G$ be a reductive group, $\pi$ be the fundamental group of a closed oriented surface of genus $g$ and $\tau$ belong to $\operatorname{Hom}(\pi, G)$. Let $Z(\tau)$ denote the centralizer of the image of $\tau$. Then the dimension of the Zariski tangent space at $\tau$ to the space $\operatorname{Hom}(\pi, G)$ is

$$
(2 g-1) \operatorname{dim}(G)+\operatorname{dim}(Z(\tau)) .
$$

Moreover, if the representation $\tau$ is irreducible then $\tau$ is a smooth point.

2.2. Irreducibility and regularity in algebraic groups. In this section, we review hypotheses needed to replace the so-called "irreducibility" and "regularity" of the case $G=\mathrm{SL}(n, \mathbb{C})$. We want to deal with semisimple or reductive groups. The natural framework is then the rather general theory of algebraic groups.

2.2.1. Reductive groups. Let $G$ be the points over $\mathbb{C}$ of a reductive algebraic group. Let $d$ be its dimension, $z$ the dimension of its center and $r+z$ the minimal dimension of the centralizer of an element in $G: r$ is the rank of the semisimple part $G / Z(G)$, or the dimension of a maximal torus included in this semisimple part.

Recall that in the cases of $\operatorname{PGL}(n, k)$ or $\operatorname{SL}(n, k)$, we have $d=n^{2}-1, z=0$, $r=n-1$.

2.2.2. Irreducibility and strong irreducibility. We recall the general notion of irreducibility, as given in [19]:

Definition 2.1 (Irreducibility). We say that $\rho \in \operatorname{Hom}(\Gamma, G)$ is irreducible if its image is not contained in any parabolic subgroup of $G$. 
Recall from [19] the following property:

Proposition 2 (Prop. 13 in [19]). If $\rho$ is irreducible, the centralizer in $G$ of $\rho(\Gamma)$ is the center of $G$.

We will need a slightly stronger notion of irreducibility known as strong irreducibility:

Definition 2.2 (Strong irreducibility). We say that $\rho \in \operatorname{Hom}(\Gamma, G)$ is strongly irreducible if the image of none of its finite index subgroups is contained in any parabolic subgroup of $G$.

Let us note that this is a quite natural and usual notion in the context of discrete subgroups of $\mathrm{SL}(n, \mathbb{C})$ or linear representations where it is rephrased as the fact that no strict subspace has a finite orbit under the group. Several appearances of this notion can be given, from divisible convex sets in [2] to random walks in groups [1] and finite group representations 9 or problems of commensurability of subgroups in the special linear group [14.

\subsubsection{Regularity.}

Definition 2.3 (Regularity). Let $H$ be a commutative group in $G$. We say that $H$ is regular if its centralizer in $G$ has the minimal dimension $r+z$.

Now let $M$ be a compact 3-manifold with boundary, and $\Gamma$ its fundamental group. As before, for each torus $T$ we fix an inclusion $\pi_{1}(T) \subset \Gamma$. We will always assume that the boundary of $M$ is not empty.

Definition 2.4 (Boundary regularity). A representation $\rho \in \operatorname{Hom}(\Gamma, G)$ is boundary regular if the image $\rho\left(\pi_{1}(T)\right)$ is regular for each torus component $T \in \partial M$.

For $G=\operatorname{PGL}(n, k)$ or $\operatorname{SL}(n, k)$, (the subgroup generated by) a single element is regular if and only if all its eigenspaces are lines. A semisimple element (in the framework of an algebraic group) is regular but a regular unipotent (as in [10]) is regular too.

\section{THE GENERAL CASE}

We may now state the general theorem:

Theorem 5. Let $G$ be a complex reductive group of dimension $d$ and rank $r$ and whose center has dimension $z$.

Let $M$ be a compact orientable 3-manifold, whose boundary is non-empty, has no sphere components, and contains $t$ torus components. Denote by $\Gamma$ its fundamental group. Let $R_{0}$ be an irreducible component of $\operatorname{Hom}(\Gamma, G)$ containing a strongly irreducible and boundary regular representation $\rho$. Let $X_{0}$ be its projection in the character variety $X(\Gamma, G)$. Then

$$
\operatorname{dim}\left(X_{0}\right) \geq r . t-d \chi(M)+z .
$$

\subsection{A general version of key lemmas.}

Lemma 6. Let $\rho$ be a strongly irreducible, boundary regular representation of $\Gamma$ in $G$ and $T$ be a torus boundary component.

Then there exists $\gamma \in \Gamma$ such that the subgroup of $G$ generated by $\rho\left(\pi_{1}(T)\right)$ and $\rho(\gamma)$ is irreducible. 
Proof. Modding out by the center of $G$, we may assume that $G$ is semisimple (the irreducibility assumption is preserved; see [19, Lemma 11]). We obtain the lemma by using results on regular elements given by Steinberg [20].

By hypothesis, the Zariski-closure $G^{\prime}$ of $\rho(\Gamma)$ is not contained in any parabolic subgroup of $G$ and neither is any of its finite index subgroups. Moreover the subgroup $H=\rho\left(\pi_{1}(T)\right)$ is a regular subgroup of $G$. From Steinberg [20, we know that it is included in a finite number of Borel subgroups of $G$. On the other hand, for each Borel subgroup, there is only a finite number of maximal parabolic subgroups of $G$ containing it [13, Section 0.8]. Hence $H$ is included in a finite number of maximal parabolic subgroups of $\mathrm{G}$.

Consider the set $A$ of elements $g \in G$ such that the subgroup generated by $g$ and $H$ is not contained in any parabolic subgroup of $G$. Its complement is the union of the finite number of maximal parabolic subgroups of $G$ containing $H$. The intersection of each of these parabolic subgroups with $G^{\prime}$ is a closed subgroup of $G^{\prime}$. Moreover, as $G^{\prime}$ is neither contained nor virtually contained in any parabolic subgroup, this closed subgroup is a proper parabolic subgroup of $G^{\prime}$ of positive codimension (it is not a finite index subgroup). Hence the complement of $A$ is Zariski-closed in $G$ and $A \cap G^{\prime}$ is the intersection of a finite number of non-empty Zariski-open subsets of $G^{\prime}$ whose complement has positive codimension.

As $G^{\prime}$ is the Zariski-closure of $\rho(\Gamma)$, there exists an element $\gamma \in \Gamma$ such that $\rho(\gamma)$ belongs to $A$. Therefore $\rho(\gamma)$ and $\rho\left(\pi_{1}(T)\right)$ are not included in any parabolic subgroup: the group they generate is irreducible.

Lemma 7. Let $\tau_{0}$ be an irreducible representation of $\pi_{1}\left(\Sigma_{2}\right)$ in $G$ and with a symplectic basis of homology $\alpha, \beta, \gamma, \delta$. Suppose $\tau_{0}(\delta)=1$ and $\tau_{0}(\langle\alpha, \beta\rangle)$ is regular.

Then the codimension in $\operatorname{Hom}\left(\pi_{1}\left(\Sigma_{2}\right), G\right)$ of the subvariety $S$ defined by $\tau(\delta)=1$ is $d-r$. Moreover, $\tau_{0}$ is a smooth point in both the subvariety $S$ and $\operatorname{Hom}\left(\pi_{1}\left(\Sigma_{2}\right), G\right)$.

Proof. By [8, Prop 1.2] reviewed before (see Theorem 4), the irreducible representation $\tau_{0}$ is a smooth point of $\operatorname{Hom}\left(\pi_{1}\left(\Sigma_{2}\right), G\right)$ and the dimension at this point is $3 d+z$.

Let us now compute the dimension of the subvariety $S$ defined by $\tau(\delta)=1$. A point in this variety is given by the image of the group generated by $\alpha, \beta$ and $\gamma$. Moreover, from the presentation of $\pi_{1}\left(\Sigma_{2}\right)$ and the condition $\tau(\delta)=1$, we get that the images of $\alpha$ and $\beta$ commute. So the dimension of $S$ is the dimension at $\left(\tau_{0}\right)_{\mid\langle\alpha, \beta\rangle}$ of the space of representations of $\mathbb{Z}^{2}$ - i.e. the fundamental group of the torus plus the dimension of the representations of $\gamma$. This gives (applying again Theorem (4) that the dimension of the Zariski-tangent subspace at $\tau_{0}$ to the subvariety $S$ is $(d+r+z)+d$. As it is the minimal possible dimension for a Zariski-tangent subspace of $S$ (by the regularity hypothesis), we get that $\tau_{0}$ is a smooth point of $S$.

We conclude that the codimension is $d-r$ as announced.

3.2. When no boundary component is a torus. We can follow step-by-step the presentation of Culler-Shalen [4] in this case. We get:

Proposition 3 (No torus boundary component). Let $M$ be a compact 3-manifold whose boundary is non-empty, and contains no sphere nor torus component. Let $G$ be a real or complex algebraic group of dimension $d$. Then for any irreducible component $R_{0}$ of $\operatorname{Hom}(\Gamma, G)$ and $X_{0}$ its projection in $X(\Gamma, G)$, we have:

$$
\operatorname{dim}\left(X_{0}\right) \geq-d \chi(M)+z .
$$


Proof. Following Remark 2.1, we want to prove $\operatorname{dim}\left(R_{0}\right) \geq-d \chi(M)+d$.

We assumed that $\partial M \neq \emptyset$. Then $M$ has the homotopy type of a finite twodimensional CW-complex with one 0-cell, $m_{1}$ 1-cells and $m_{2}$ 2-cells. The Euler characteristic of $M$ is then $1-m_{1}+m_{2}$.

This decomposition gives a presentation

$$
\Gamma=\left\langle g_{1}, \ldots, g_{m_{1}} \mid r_{1}, \ldots, r_{m_{2}}\right\rangle .
$$

In other words, $\operatorname{Hom}(\Gamma, G)$ is the preimage under a regular map from $G^{m_{1}}$ to $G^{m_{2}}$ of the point $(1, \ldots, 1) \in G^{m_{2}}$. As an application of Proposition 1 the dimension of any irreducible component - e.g. $R_{0}$ - is greater than $d \cdot\left(m_{1}-m_{2}\right)=-d \chi(M)+d$.

3.3. Conclusion. Replacing both lemmas of the proof in the simple case by their general version above, replacing $n-1$ by $r$ and $n^{2}-1$ by $d$, we readily get the announced lower bound:

$$
\operatorname{dim}\left(X_{0}\right) \geq r . t-d \chi(M)+z .
$$

\section{ACKNOWLEDGEMENT}

We thank Y. Benoist, P. Dingoyan, S. Diverio, P.-V. Koseleff, F. Rouillier, P. Will and M. Thistlethwaite for many discussions.

\section{REFERENCES}

[1] CURVE. http://curve.unhyperbolic.org/.

[2] Yves Benoist, Convexes divisibles. III (French, with English and French summaries), Ann. Sci. École Norm. Sup. (4) 38 (2005), no. 5, 793-832, DOI 10.1016/j.ansens.2005.07.004. MR2195260

[3] Nicolas Bergeron, Elisha Falbel, Antonin Guilloux, Pierre-Vincent Koseleff, and Fabrice Rouillier, Local rigidity for $\mathrm{PGL}(3, \mathbb{C})$-representations of 3-manifold groups, Exp. Math. 22 (2013), no. 4, 410-420, DOI 10.1080/10586458.2013.832441. MR3171102

[4] Marc Culler and Peter B. Shalen, Varieties of group representations and splittings of 3manifolds, Ann. of Math. (2) 117 (1983), no. 1, 109-146, DOI 10.2307/2006973. MR683804

[5] Elisha Falbel, A spherical CR structure on the complement of the figure eight knot with discrete holonomy, J. Differential Geom. 79 (2008), no. 1, 69-110. MR2401419

[6] E. Falbel, A. Guilloux, P.-V. Koseleff, F. Rouillier, and M. Thistlethwaite, Character varieties for $\operatorname{SL}(\mathbf{3}, \mathbb{C})$ : the figure eight knot, Exp. Math. 25 (2016), no. 2, 219-235, DOI 10.1080/10586458.2015.1068249. MR3463570

[7] E. Falbel, P.-V. Koseleff, and F. Rouillier, Representations of fundamental groups of 3manifolds into PGL $(3, \mathbb{C})$ : exact computations in low complexity, Geom. Dedicata 177 (2015), 229-255, DOI 10.1007/s10711-014-9987-x. MR3370032

[8] William M. Goldman, The symplectic nature of fundamental groups of surfaces, Adv. in Math. 54 (1984), no. 2, 200-225, DOI 10.1016/0001-8708(84)90040-9. MR.762512

[9] Benedict H. Gross, Group representations and lattices, J. Amer. Math. Soc. 3 (1990), no. 4, 929-960, DOI 10.2307/1990907. MR1071117

[10] Antonin Guilloux, Deformation of hyperbolic manifolds in $\operatorname{PGL}(n, \mathbf{C})$ and discreteness of the peripheral representations, Proc. Amer. Math. Soc. 143 (2015), no. 5, 2215-2226, DOI 10.1090/S0002-9939-2014-12376-8. MR 3314127

[11] Yves Guivarc'h and Albert Raugi, Propriétés de contraction d'un semi-groupe de matrices inversibles. Coefficients de Liapunoff d'un produit de matrices aléatoires indépendantes (French, with English summary), Israel J. Math. 65 (1989), no. 2, 165-196, DOI 10.1007/BF02764859. MR998669

[12] M. Heusener, V. Munoz, and J. Porti, The sl(3,c)-character variety of the figure eight knot, arXiv:1505.04451 (2015).

[13] James E. Humphreys, Conjugacy classes in semisimple algebraic groups, Mathematical Surveys and Monographs, vol. 43, American Mathematical Society, Providence, RI, 1995. MR.1343976 
[14] D. D. Long and A. W. Reid, Commensurability and the character variety, Math. Res. Lett. 6 (1999), no. 5-6, 581-591, DOI 10.4310/MRL.1999.v6.n5.a11. MR.1739217

[15] Pere Menal-Ferrer and Joan Porti, Local coordinates for $\operatorname{SL}(n, \mathbf{C})$-character varieties of finitevolume hyperbolic 3-manifolds (English, with English and French summaries), Ann. Math. Blaise Pascal 19 (2012), no. 1, 107-122, DOI 10.5802/ambp.306. MR2978315

[16] David Mumford, Algebraic geometry. I, Springer-Verlag, Berlin-New York, 1976. Complex projective varieties; Grundlehren der Mathematischen Wissenschaften, No. 221. MR 0453732

[17] Walter D. Neumann and Don Zagier, Volumes of hyperbolic three-manifolds, Topology 24 (1985), no. 3, 307-332, DOI 10.1016/0040-9383(85)90004-7. MR815482

[18] R. W. Richardson, Commuting varieties of semisimple Lie algebras and algebraic groups, Compositio Math. 38 (1979), no. 3, 311-327. MR535074

[19] Adam S. Sikora, Character varieties, Trans. Amer. Math. Soc. 364 (2012), no. 10, 5173-5208, DOI 10.1090/S0002-9947-2012-05448-1. MR2931326

[20] Robert Steinberg, Regular elements of semisimple algebraic groups, Inst. Hautes Études Sci. Publ. Math. 25 (1965), 49-80. MR0180554

[21] W. Thurston, The geometry and topology of 3-manifolds (1980). http://library.msri.org/ books $/ \mathrm{gt} 3 \mathrm{~m} /$.

[22] J. Tits, Free subgroups in linear groups, J. Algebra 20 (1972), 250-270. MR0286898

[23] André Weil, On discrete subgroups of Lie groups, Ann. of Math. (2) 72 (1960), 369-384. MR0137792

Institut de Mathématiques de Jussieu-Paris Rive Gauche, Unité Mixte de Recherche 7586 DU CNRS, CNRS UMR 7586

E-mail address: elisha.falbel@imj-prg.fr

INRIA EPI-OURAGAN, Université Pierre et Marie Curie, 4 Place Jussieu 75252 Paris Cédex, France - and - Institut de Mathématiques de Jussieu-Paris Rive Gauche, Université Pierre et Marie Curie, 4 Place Jussieu, 75252 Paris Cédex, France

E-mail address: antonin.guilloux@imj-prg.fr 